\title{
POEDEIRAS ALIMENTADAS COM DIFERENTES NÍVEIS DE ENERGIA E OLEO DE SOJA NA RAÇÃO*
}

\author{
LAYERS FED WITH DIFFERENT LEVELS OF ENERGY AND SOYBEAN OIL IN FEED
}

\author{
Costa, F.G.P. ${ }^{1}$, B.J.S. Quirino ${ }^{1}$, P.E.N. Givisiez ${ }^{1}$, J.H.V. Silva ${ }^{2}$, H.H.S. Almeida ${ }^{3}$, J.S. Costa ${ }^{3}$, \\ C.F.S. Oliveira ${ }^{3}$ e C.C. Goulart ${ }^{1,4}$
}

1'Departamento de Zootecnia. UFPB. Areia-PB. 58397-000. Brasil. fperazzo@cca.ufpb.br

${ }^{2}$ Departamento de Agropecuária. UFPB. Bananeiras-PB. Brasil.

${ }^{3} \mathrm{PIBIC/CNPq}$. Areia-PB. Brasil.

${ }^{4}$ UVA-Sobral-CE. Brasil.

\section{PalaVRas ChaVe ADICIONAIS \\ Conversão energética. Postura. Qualidade de ovo.}

\section{RESUMO}

Objetivou-se avaliar os efeitos de níveis crescentes de energia e de óleo de soja sobre o desempenho e a qualidade interna e externa dos ovos de poedeiras semipesadas. Foram utilizadas 360 poedeiras comerciais da linhagem Bovans Goldline, com 29 semanas de idade, durante cinco períodos de 28 dias, distribuídas num delineamento inteiramente casualizado, em esquema fatorial $3 x$ 3 , totalizando 9 tratamentos com 5 repetições de oito aves cada. Os fatores estudados foram níveis de óleo de soja (1, 2 e 3\%) e níveis de energia metabolizável na ração $(2600,2750$ e $2900 \mathrm{kcal} /$ $\mathrm{kg}$ ). Não foi observada interação entre os níveis do óleo de soja e a energia metabolizável da dieta. Os níveis de óleo de soja não promoveram efeito para nenhum dos parâmetros avaliados. Os níveis de energia metabolizável não influenciaram a produção de ovos, conversão alimentar, massa de ovos, peso de gema e de casca, percentagem de gema e de clara e gravidade específica. Foram observadas diferenças para conversão energética, peso do ovo e de clara, e percentagem de casca, sendo os maiores pesos observados no menor nível de energia e a maior percentagem observada no maior nível de energia. O fornecimento de $2600 \mathrm{kcal}$ de EM/kg foi mais adequado para manter a produção e o peso corporal e para promover melhoria na conversão energética e aumento no peso do ovo.

*Parte da Dissertação de Mestrado da segunda autora.

Recibido: 2-9-07. Aceptado: 14-5-08.

\author{
AdDitiOnAL KEYWORDS \\ Energetic conversion. Laying. Egg quality.
}

\section{SUMMARY}

The aim of this work was to evaluate increasing levels of energy and soybean oil on performance and internal and external egg quality. Three hundred and sixty semi-heavy laying hens Bovans Goldline, 29 week-old, were used, during five periods of 28 days each. It was used a completely randomized design, in a double factorial $(3 \times 3)$, totaling nine treatments, with five replicates of eight birds each. Studied factors were soybean oil $(1,2$ and $3 \%)$ and energy $(2600,2750$ and 2900 kcal EMkg-1) levels on diet. No interaction was observed among soybean oil and metabolizable energy. Soybean oil did not promote effect over none of the evaluated parameters. No effect was observed for energy levels on egg production, feed conversion, egg mass, yolk and shell weight, yolk and albumen percentage and specific gravity. Differences were observed for feed conversion, egg and albumen weight and shell percentage, given that the highest weights were observed at the lowest energy level and the highest percentage was observed at the highest energy level. Providing $2600 \mathrm{kcal} \mathrm{EMkg}^{-1}$ was more suitable in order to keep production and body weight and also to promote improvement on energetic conversion and an increase on egg weight.

\section{INTRODUÇÃO}

A avicultura de postura tem evoluído muito nos últimos anos e, como segmento

Arch. Zootec. 58 (223): 405-411. 2009. 
importante na produção de alimento humano de alto valor biológico, tem se adequado às técnicas que possibilitam a melhoria da eficiência de produção das aves. A alimentação dessas aves representa a maior fração do custo de produção e pequenas melhorias na eficiência de utilização dos nutrientes das rações podem resultar em grandes economias (Rodrigues et al., 2005).

$\mathrm{Na}$ exploração de poedeiras, vários aspectos podem alterar o desempenho e a qualidade dos ovos, verificando que a nutrição é um dos principais pontos críticos no crescimento, desenvolvimento e produtividade dessas aves. As rações das aves são formuladas de acordo com a quantidade de nutrientes requeridos para realizarem as funções básicas do organismo e as produtivas de forma mais eficiente. Porém, essas exigências não são constantes e variam com a idade, sexo, ambiente, níveis de energia e aminoácidos da ração, dentre outros fatores. Por isso, é necessário fazer avaliações periódicas dos níveis de nutrientes adequados para cada região (Costa et al., 2004).

Apesar das constantes pesquisas sobre o uso de alimentos não convencionais em rações de aves, as formulações ainda são basicamente com milho e farelo de soja, principais fontes protéica e energética. No entanto, para melhor balanceamento energético, é necessária a inclusão de fontes lipídicas nas rações (Rodrigues et al., 2005). O National Research Council (NRC, 1994) destaca a melhora na palatabilidade e na conversão alimentar e a redução na perda de nutrientes, entre outros, como efeitos benéficos do uso de gorduras nas formulações.

No Brasil, em decorrência de a maioria dos galpões utilizados serem abertos, observam-se grandes variações de temperatura ambiente interna, alterando, portanto, as exigências de energia metabolizável das aves.

Rosa et al. (1997) afirma que as exigências nutricionais das aves expressas nos manuais que as acompanham, na maioria das vezes são determinadas em condições ambientais diferentes daquelas em que serão criadas.

O nível de energia é normalmente utilizado como um ponto de partida para a formulação das dietas, servindo de base para a fixação dos níveis de outros nutrientes como proteína bruta e/ou aminoácidos, ácidos graxos e minerais. A exigência de energia das poedeiras está condicionada a fatores como peso corporal, ganho de peso, produção de massa de ovos, nível de empenamento, temperatura ambiente, composição corporal e do ovo e eficiência de utilização dos nutrientes da dieta para deposição em tecido corporal e em ovo (Faria e Santos, 2005).

Atualmente, os óleos são utilizados rotineiramente na alimentação de aves com a finalidade de aumentar a energia das rações, melhorar a digestão e a absorção de constituintes não lipídicos e aumentar o tempo de retenção dos alimentos, além de fonte de ácidos graxos para obtenção de produtos com perfil nutricional diferenciado (Santos, 2005).

O objetivo deste estudo foi avaliar diferentes níveis de energia e de óleo de soja sobre o desempenho e a qualidade de ovo de poedeiras comerciais no período de postura.

\section{MATERIAL E MÉTODOS}

O experimento foi realizado no setor de Avicultura do Departamento de Zootecnia da Universidade Federal da Paraíba, na cidade de Areia-PB. A referida cidade encontra-se nas coordenadas: $6^{\circ} 58^{\prime} 554^{\prime \prime} \mathrm{de}$ latitude sul e $35^{\circ} 43^{\prime} 047^{\prime \prime}$ de longitude oeste e a $618 \mathrm{~m}$ da altitude acima do nível do mar. Os valores médios de temperatura máxima, mínima, média, umidade relativa do ar e precipitação registradas no período experimental foram respectivamente 27,$4 ; 21,4 \mathrm{e}$ $23,6^{\circ} \mathrm{C} ; 86 \%$ e $138,4 \mathrm{~mm}$.

O delineamento experimental utilizado foi o inteiramente casualizado em esquema fatorial duplo $(3 \times 3)$, (níveis de óleo x níveis 


\section{POEDEIRAS ALIMENTADAS COM DIFERENTES NÍVEIS DE ENERGIA E ÓLEO SOJA}

de energia), totalizando 9 tratamentos: T1 (1\% e 2600), T2 (1\% e 2750), T3 ( $1 \%$ e 2900$)$ T4 (2\% e 2600$),$ T5 (2\% e 2750$),$ T6 $(2 \%$ e $2900)$, T7 (3\% e 2600$),$ T 8 ( $3 \%$ e 2750$)$ e T9 (3\% e 2900$)$ com 5 repetições e 8 aves cada, como pode ser observado na tabela $\mathbf{I}$.

Foram utilizadas 360 poedeiras comer- ciais da linhagem Bovans Goldline, com 29 semanas de idade, alojadas em galpão convencional de postura, com comedouros tipo calha e bebedouros tipo nipple. As aves passaram por um período de adaptação às rações avaliadas por sete dias, iniciando o experimento logo a seguir. O peso médio

Tabela I. Composição percentual e valores calculados das dietas ${ }^{1}$. (Percentual composition and calculated dietary values).

\begin{tabular}{|c|c|c|c|c|c|c|c|c|c|}
\hline Ingredientes & T1 & T2 & T 3 & T 4 & T 5 & T 6 & T7 7 & T 8 & T9 \\
\hline Milho & 58,988 & 64,009 & 68,5 & 56,047 & 61,068 & 66,091 & 53,106 & 58,127 & 63,14 \\
\hline Farelo de soja (46\%) & 21,400 & 20,462 & 17,229 & 21,943 & 21,016 & 20,061 & 22,486 & 21,559 & 20,627 \\
\hline Milho farelo glúten (60\%) & 0,000 & 0,000 & 1,748 & 0,000 & 0,000 & 0,000 & 0,000 & 0,000 & 0,000 \\
\hline Óleo de soja & 1,000 & 1,000 & 1,000 & 2,000 & 2,000 & 2,000 & 3,000 & 3,000 & 3,000 \\
\hline Calcário & 9,361 & 9,373 & 9,395 & 9,354 & 9,366 & 9,377 & 9,347 & 9,359 & 9,371 \\
\hline Fosfato bicálcico & 1,287 & 1,275 & 1,274 & 1,294 & 1,282 & 1,270 & 1,302 & 1,289 & 1,277 \\
\hline Sal & 0,423 & 0,421 & 0,423 & 0,424 & 0,422 & 0,420 & 0,425 & 0,423 & 0,421 \\
\hline Mistura mineral $^{2}$ & 0,050 & 0,050 & 0,050 & 0,050 & 0,050 & 0,050 & 0,050 & 0,050 & 0,050 \\
\hline Mistura vitamínica ${ }^{3}$ & 0,025 & 0,025 & 0,025 & 0,025 & 0,025 & 0,025 & 0,025 & 0,025 & 0,025 \\
\hline a HCL & 0,000 & 0,006 & 0,063 & 0,000 & 0,000 & 0,013 & 0,000 & 0,000 & 0,003 \\
\hline DL-m & 0,172 & 0,165 & 0,160 & 0,176 & 0,169 & 0,162 & 0,180 & 0,173 & 0,166 \\
\hline colina & 0,070 & 0,070 & 0,070 & 0,070 & 0,070 & 0,070 & 0,070 & 0,0 & 0,070 \\
\hline Etoxiq & 0,010 & 0,010 & 0,010 & 0,010 & 0,010 & 0,010 & 0,010 & 0,010 & 0,010 \\
\hline Areia lavada (inerte) & 7,214 & 3,135 & 0,010 & 8,607 & ,522 & 0,449 & 999 & 15 & 1,832 \\
\hline Total & 100,0 & 100,0 & 100,0 & 100,0 & 100,0 & 100,0 & 100,0 & 100,0 & 100,0 \\
\hline \multicolumn{10}{|l|}{ Composição calculada } \\
\hline EM (kcal/kg) & 2600 & 2750 & 2900 & 2600 & 2750 & 2900 & 2600 & 2750 & 2900 \\
\hline (\%) & 15,000 & 15,000 & 15,000 & 15,000 & 15,000 & 15,000 & 15,000 & 15,000 & 15,000 \\
\hline Cálcio (\%) & 4,000 & 4,000 & 4,000 & 4,000 & 4,000 & 4,000 & 4,000 & 4,000 & 4,000 \\
\hline Fósforo disponível (\%) & 0,326 & 0,326 & 0,326 & 0,326 & 0,326 & 0,326 & 0,326 & 0,326 & 0,326 \\
\hline Lisina digestível (\%) & 0,661 & 0,652 & 0,640 & 0,669 & 0,656 & 0,652 & 0,677 & 0,663 & 0,652 \\
\hline Metionina digest. (\%) & 0,387 & 0,383 & 0,390 & 0,390 & 0,386 & 0,381 & 0,393 & 0,388 & 0,384 \\
\hline Met + cist digest. (\%) & 0,600 & 0,599 & 0,613 & 0,600 & 0,599 & 0,599 & 0,601 & 0,600 & 0,599 \\
\hline Arginina digestível & 0,887 & 0,876 & 0,823 & 0,894 & 0,882 & 0,871 & 0,900 & 0,889 & 0,878 \\
\hline Treonina digestível (\%) & 0,498 & 0,497 & 0,492 & 0,498 & 0,497 & 0,496 & 0,499 & 0,498 & 0,497 \\
\hline Cloro $(\%)$ & 0,284 & 0,285 & 0,289 & 0,283 & 0,284 & 0,285 & 0,282 & 0,284 & 0,285 \\
\hline Potássio (\%) & 0,571 & 0,569 & 0,526 & 0,572 & 0,570 & 0,567 & 0,573 & 0,571 & 0,569 \\
\hline Sódio (\%) & 0,200 & 0,200 & 0,200 & 0,200 & 0,200 & 0,200 & 0,200 & 0,200 & 0,200 \\
\hline
\end{tabular}

'Valores calculados de acordo com Rostagno et al. (2000).

${ }^{2}$ Composição/kg: manganês: 160g; ferro: 100 g; zinco: 100 g; cobre: 20 g; cobalto: 2 g; iodo: 2 g, excipiente: q.s.p. $1000 \mathrm{~g}$.

${ }^{3}$ Composição/kg: vit. A 12000000 U.I.; vit $D_{3} 3600000$ U.I.; vit. E 3500 U.I.; vit $B_{1} 2,5$ g; vit $B_{2} 8$ g; vit $B_{6}$ $5 \mathrm{~g}$; ác. pantotênico $12 \mathrm{~g}$; biotina $200 \mathrm{mg}$; vit. K $3 \mathrm{~g}$; ác. fólico $1,5 \mathrm{~g}$; ác. nicotínico $40 \mathrm{~g}$; vit. $\mathrm{B}_{12} 20 \mathrm{~g}$; selênio $150 \mathrm{mg}$; excipiente q.s.p. $1000 \mathrm{~g}$.

${ }^{4}$ Antioxidante. 
inicial foi de $1,855 \mathrm{~g}$. O período experimental foi dividido em 5 períodos de 28 dias cada.

O programa de luz adotado foi o de luz natural, água fornecida ad libitum e as rações experimentais foram fornecidas na quantidade de $120 \mathrm{~g} /$ ave/dia. As dietas à base de milho e farelo de soja foram formuladas segundo as recomendações de Rostagno et al. (2000), exceto em relação aos níveis de energia utilizados.

Os parâmetros avaliados foram: ganho de peso final (g), massa de ovos (g/ave/dia); conversão energética por dúzia (kcal de $\mathrm{EM} / \mathrm{dz}$ ) e massa de ovos (kcal de EM/g); conversão alimentar por dúzia de ovos $(\mathrm{kg} /$ dz) e massa de ovos; produção de ovos (\%/ ave/dia); peso do ovo, gema, clara e casca $(\mathrm{g})$; percentagem de gema, clara e casca $(\%)$; e gravidade específica.

Os valores das exigências de energia e de óleo foram estimados através da análise das variáveis pelo modelo de regressão, utilizando o programa SAS (2000).

\section{RESULTADOSEDISCUSSÃO}

Nao houve interação entre os fatores óleo de soja e energia mostrando assim, que um fator não interferiu no outro e, portanto seus efeitos foram estudados separadamente. As análises de variância e de regressão dos parâmentros de desempenho e qualidade de ovo em relação fatores óleo de soja e energia metabolizável encontram-se na tabela II, sendo os mesmos representados pelos seus valores médios.

Os níveis de óleo de soja não promoveram efeito para nenhum dos parâmetros avaliados. A inclusão de até 3\% de óleo de soja não foi suficiente para promover aumento na conversão energética e, portanto não houve melhora significativa no aproveitamento dos nutrientes a ponto de aumentar a produção de ovos ou o peso do ovo e de seus constituintes (casca, gema e clara). Estes resultados corroboram em parte os de Rodrigues et al. (2005), os quais avaliaram níveis crescentes de óleo de soja $(0,1,2,3$ e $4 \%$ ) no desempenho de poedeiras de segundo ciclo e não observaram diferenças no consumo de ração, massa de ovos, peso dos ovos, percentagem e espessura de casca e a gravidade específica.

Santos (2005) trabalhando com poedeiras Hy-line w-36 de 23 a 42 semanas de idade observou que a inclusão do óleo de soja nas dietas não possibilitou melhora na percentagem de ovos, conversão alimentar, massa de ovo, ganho de peso corporal, peso dos ovos e nas percentagens da gema, clara e casca.

Muramatsu et al. (2005) utilizando poedeiras com 35 semanas de idade submetidas à adição de três níveis de óleo de soja na ração com milho $(3,3 \% ; 4,3 \%$ e $5,3 \%)$ e três níveis na ração com milheto $(2,5 \% ; 3,5 \%$ e $4,5 \%)$, também não obtiveram efeito significativo sobre a qualidade externa do ovo, a porcentagem e espessura da casca, peso da clara, peso da gema, percentagem da clara e percentagem da gema.

Em um experimento com poedeiras alimentadas com rações suplementadas com óleo de algodão, óleo de soja, óleo de girassol e óleo de canola, Filardi et al. (2004) não verificaram diferenças significativas na produção de ovos. Da mesma forma, Murata (1998) avaliando a inclusão de 3\% dos óleos de soja e canola e peixe na dieta de galinhas poedeiras comerciais, com 49 e 38 semanas de idade, respectivamente, constatou que não houve alteração no desempenho zootécnico.

Shafey et al. (1992) observaram que o óleo de soja em rações de poedeiras comerciais ocasionou aumento na percentagem de ovos, mas não afetou significativamente o consumo de ração, o peso da gema e do ovo.

Não foi observado efeito dos níveis de energia na produção de ovos, conversão alimentar, massa de ovos, peso da gema e da casca, percentagem de gema e de clara, gravidade específica e ganho de peso.

O valor médio obtido para gravidade específica $(1,085)$ foi semelhante ao de 


\section{POEDEIRAS ALIMENTADAS COM DIFERENTES NÍVEIS DE ENERGIA E ÓLEO SOJA}

Freitas et al. (2004) que compararam métodos de determinação da gravidade específica e encontraram o valor médio de $1,086 \mathrm{~g} /$ $\mathrm{cm}^{3}$ e peso médio do ovo igual a $58,5 \mathrm{~g}$. O peso médio do ovo obtido no presente experimento foi $68,15 \mathrm{~g}$.

Quanto à produção de ovos, Pecuri e Coon (1991) também não encontraram diferenças quando trabalharam com dietas com nível energético variando de 2645 a $2971 \mathrm{kcal}$ de $\mathrm{EM} / \mathrm{kg}$, e ainda afirmaram que a eficiência de utilização da energia do alimento para a produção de ovos pode diminuir conforme se aumenta a densidade energética da dieta. Por outro lado, Latshaw et al. (1990), trabalhando com níveis energéti- cos de 2490 a $3000 \mathrm{kcal} \mathrm{EM} / \mathrm{kg}$, obtiveram a maior produção em níveis intermediários.

Foram observadas diferenças na conversão energética por dúzia e por massa de ovo, peso do ovo e da clara, e percentagem de casca. Observou-se piora na conversão energética à medida que aumentou o nível energético da ração. O menor nível energético $(2600 \mathrm{EM} / \mathrm{kg})$ foi o que promoveu o melhor aproveitamento da energia e, por conseqüência, dos nutrientes fornecidos; dessa maneira, promoverem aumento no peso do ovo devido ao aumento no peso da clara.

Embora o consumo de energia nos diversos tratamentos não tenha influenciado o

Tabela II. Valores médios de produção de ovos (PRO); conversão alimentar por dúzia (CADZ) e por massa de ovos (CAMO); conversão energética por dúzia (CEDZ, kcal de EM/ $d z)$ e por massa de ovos (CEMO, kcal de EM/g); massa de ovos (MO); peso do ovo (PO), gema $(P G)$, clara $(P C L)$ e casca (PCA); percentagem de gema $(G)$, clara $(\% C L)$ e casca $(\% C A S)$ e gravidade específica (GRES); em relação aos níveis de energia metabolizável e de óleo de soja avaliados. (Mean values of egg production (PRO); feed conversion per dozen (CADZ) and per egg mass (CAMO)); energetic conversion per dozen (CEDZ, kcal ME/dz) and per egg mass (CEMO, kcal $\mathrm{ME} / \mathrm{g})$; egg mass (MO); egg (PO), yolk (PG), albumen (PCL) and eggshell (PCA) weights; yolk $(\% G)$, albumen (\%CL) and eggshell (\%CAS) and percentage specific gravity (GRES) in relation to metabolizable energy and soybean oil evaluates).

\begin{tabular}{|c|c|c|c|c|c|c|c|c|c|c|c|}
\hline \multicolumn{6}{|c|}{ Níveis de energia metabolizável } & \multicolumn{3}{|c|}{ Níveis de óleo de soja } & \multirow[b]{2}{*}{ RG } & \multirow[b]{2}{*}{ Média } & \multirow[b]{2}{*}{ CV \% } \\
\hline & 2600 & 2750 & 2900 & RG & $\mathrm{R}^{2}$ & $1 \%$ & $2 \%$ & $3 \%$ & & & \\
\hline \multicolumn{12}{|l|}{ Variáveis } \\
\hline PRO (\%) & ) 86,96 & 88,05 & 89,07 & ns & - & 88,39 & 87,46 & 88,22 & ns & $88,39 \pm 4,53$ & 5,14 \\
\hline CADZ & 2,08 & 2,05 & 2,03 & ns & - & 2,04 & 2,07 & 2,05 & ns & $2,05 \pm 0,11$ & 5,52 \\
\hline CAMO & 2,00 & 2,00 & 2,01 & ns & - & 2,00 & 2,01 & 2,00 & ns & $2,00 \pm 0,10$ & 4,78 \\
\hline CEDZ & 4315,99 & 4496,33 & 4688,05 & $* *$ & 0,99 & 4487,74 & 4523,88 & 4488,75 & $\mathrm{~ns}$ & $4500,12 \pm 236,51$ & 5,26 \\
\hline CEMO & 4153,77 & 4395,11 & 4648,93 & ** & 0,99 & 4406,13 & 4410,92 & 4380,76 & ns & $4399,27 \pm 214,63$ & 4,88 \\
\hline MO (g) & 60,05 & 59,99 & 59,79 & ns & - & 60,10 & 59,53 & 60,19 & ns & $59,94 \pm 2,88$ & 4,80 \\
\hline PO (g) & 69,17 & 68,15 & 67,14 & ${ }^{* *}$ & 0,97 & 68,04 & 68,19 & 68,24 & ns & $68,15 \pm 2,34$ & 3,43 \\
\hline PG (g) & 17,59 & 17,35 & 17,16 & ns & & 17,39 & 17,37 & 17,33 & $\mathrm{~ns}$ & $17,36 \pm 0,69$ & 3,98 \\
\hline PCL (g) & 42,68 & 41,95 & 41,25 & * & 0,91 & 41,77 & 41,97 & 42,14 & ns & $41,96 \pm 1,78$ & 4,24 \\
\hline PCA (g) & 6,45 & 6,42 & 6,38 & ns & - & 6,41 & 6,45 & 6,39 & ns & $6,42 \pm 0,22$ & 3,49 \\
\hline$\% \mathrm{G}$ & 25,47 & 25,51 & 25,59 & ns & - & 25,60 & 25,52 & 25,45 & ns & $25,53 \pm 0,63$ & 2,47 \\
\hline$\% \mathrm{CL}$ & 61,62 & 61,47 & 61,37 & ns & - & 61,27 & 61,49 & 61,71 & ns & $61,49 \pm 0,86$ & 1,40 \\
\hline$\%$ CAS & 8,28 & 8,40 & 8,43 & $* \star$ & 0,90 & 8,36 & 8,41 & 8,35 & ns & $8,37 \pm 0,15$ & 1,84 \\
\hline GRES & 1,085 & 1,085 & 1,085 & ns & - & 1,085 & 1,086 & 1,085 & ns & $1,085 \pm 0,001$ & 0,13 \\
\hline
\end{tabular}

RG: regressão; CV: coeficiente de variação. ns: $p>0,05 ;{ }^{*} p<0,05 ;{ }^{* *} p<0,01$, pelo Teste $F$. 


\section{COSTA, QUIRINO, GIVISIEZ, SILVA, ALMEIDA, COSTA, OLIVEIRAE GOULART}

peso corporal e a produção de ovos, os menores consumos observados pelo fornecimento controlado da ração (120 g/ave/ dia) podem ter promovido melhor conversão energética, indicando melhor eficiência do aproveitamento da energia da dieta. Resultados contrários foram observados por Xavier e Peixoto (1997) que verificaram efeito linear crescente para o peso dos ovos à medida que foi aumentada a concentração energética da dieta.

\section{BIBLIOGRAFIA}

Costa, F.G.P., H.C. Souza, C.A.V. Gomes, L.R. Barros, P.A. Brandão, G.A.J. Nascimento, A.W.R. Santos e V.S.A. Junior. 2004. Níveis de proteína bruta e energia metaboilizável na produção e qualidade dos ovos de poedeiras da linhagem Lohmann Brown. Ciênc. Agrotec., 28: 1421-1427.

Faria, D.E. e A.L. Santos. 2005. Exigências nutricionais de galinhas poedeiras. Em: Simpósio Internacional sobre Exigências Nutricionais de Aves e Suínos. Viçosa. Anais...Viçosa. MG. p. 229-315.

Filardi, R.S., O.M. Junqueira, A.C. Laurentiz, E.M. Casartelli, K.F. Duarte e E.A. Rodrigues. 2004. Efeito de diferentes fontes de gordura sobre o desempenho, qualidade e perfil lipídico dos ovos de poedeiras vermelhas em segundo ciclo. Em: Conferência de Ciência e Tecnologia Avícola. Anais ... Associação Brasileira de Produtores de Pintos de Corte. Santos, SP. p. 30.

Freitas, E.R., N.K. Sakomura, M.M. Gonzales e N.A.A. Barbosa. 2004. Comparação de métodos de determinação da gravidade específica de ovos de poedeiras comerciais. Pesqui. Agropecu. Bras., 39: 509-512.

Lastshaw, J.D., G.B. Havenstein and V.D. Tolle. 1990. Energy level in the laying diet and its effects on the perfomance of three commercial leghorn strains. Poultry Sci., 69: 1998-2007.

Muramatsu, K., J.H. Stringhini, M.B. Café, R.M. Jardim Filho, L. Andrade e F. Godoi. 2005. Desempenho, qualidade e composição de ácidos graxos do ovo de poedeiras comerciais alimentadas com rações formuladas com milho ou milheto contendo diferentes níveis de óleo
O aumento da percentagem de casca à medida em que aumentou o nível de energia utilizado foi provavelmente devido à redução do peso do ovo e consequentemente peso da casca.

\section{CONCLUSÃO}

O fornecimento de $2600 \mathrm{EM} / \mathrm{kg}$ foi o nível mais adequado para manter a produção e para promover melhoria na conversão energética e aumento no peso do ovo.

vegetal. Acta Sci. Anim. Sci., 27: 43-48.

Murata, L.S. 1998. Efeito de fontes de óleo da ração sobre o desempenho e o perfil lipídico dos ovo e sangue de poedeiras comerciais. Tese (Doutorado). Faculdade de Ciências Agrárias e Veterinárias, Universidade Estadual Paulista. São Paulo. 66 p.

NRC. 1994. Nutrient requirements of poultry. $9^{\text {th }}$ ed. National Academy Press. Washington, DC.

Pecuri, A. and C. Coon. 1991. Effect of temperature and dietary energy on layer perfomance. Poultry Sci., 70: 126-138.

Rodrigues, E.A., L.C. Cancherini, O.M. Junqueira, A.C. Laurentiz, R.S. Filardi, K.F. Duarte e E.M. Casarelli. 2005. Desempenho, qualidade da casca e perfil lipídico de gemas de ovos de poedeiras comerciais alimentadas com níveis crescentes de óleo de soja no segundo ciclo de postura. Acta Sci. Anim. Sci., 27: 207-212.

Rosa, A.P., I. Zanella, J. Their e N.S. Vieira. 1997 Influência de diferentes níveis de proteína bruta e energia metabolizável no desempenho de poedeiras Rhode Island Red na fase de recria. Rev. Bras. Zootecn., 26: 159-163.

Rostagno, H.S., L.F.T. Albino, J.L. Donzele, P.C. Gomes, A.S. Ferreira, R.F. Oliveira e D.C. Lopes. 2000. Composição de alimentos exigências nutricionais de aves e suínos: Tabelas brasileiras de aves e suínos. UFV. Viçosa-MG. 141 p.

Santos, M.S.V. 2005. Avaliação do desempenho e qualidade dos ovos de poedeiras comerciais, submetidas às dietas suplementadas com diferentes óleos vegetais. Fortaleza. Tese (Doutorado). Universidade Federal do Ceará. 174 p. SAS. 2000. SAS User's guide: Statistics. Version

Archivos de zootecnia vol. 58, núm. 223, p. 410. 


\section{POEDEIRAS ALIMENTADAS COM DIFERENTES NÍVEIS DE ENERGIA E ÓLEO SOJA}

8. SAS Institute Inc. Cary, NC.

Shafey, T.M., J.G. Dingle and M.W. McDonald. 1992. Comparison between wheat, triticale, rye, soybean oil and strain of laying bird on the production, and cholesterol and fatty acid contents of eggs. Brit. Poultry Sci., 33:
339-346.

Xavier, E.G. e R.R. Peixoto. 1997. Nível de energia metabolizável em rações para poedeiras nas condições de temperatura e umidade relativa no inverno do extremo sul do Brasil. Rev. Bras. Zootecn., 26: 364-374. 